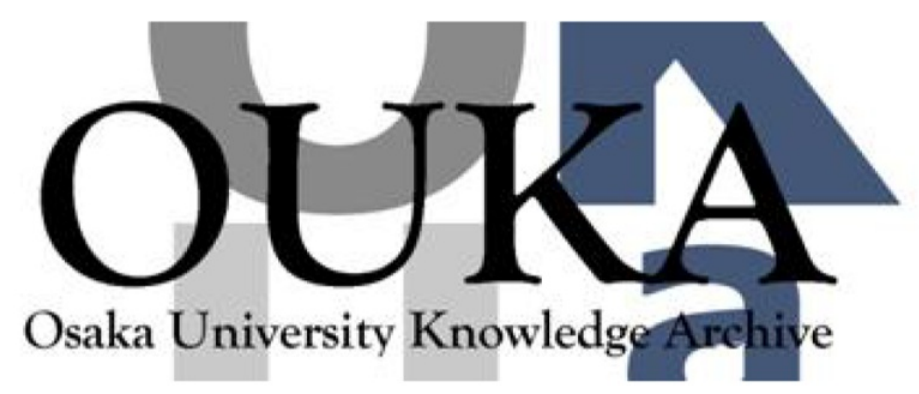

\begin{tabular}{|c|l|}
\hline Title & $\begin{array}{l}\text { Image reconstruction for thin observation } \\
\text { module by bound optics by using the iterative } \\
\text { backprojection method }\end{array}$ \\
\hline Author(s) & $\begin{array}{l}\text { Nitta, Kouichi; Shogenji, Rui; Miyatake, } \\
\text { Shigehiro et al. }\end{array}$ \\
\hline Citation & Applied Optics. 45(13) p. 2893-p. 2900 \\
\hline Issue Date & $2006-05-01$ \\
\hline oaire:version & VoR \\
\hline URL & https://hdl. handle.net/11094/2900 \\
\hline rights & \\
\hline Note & \\
\hline
\end{tabular}

Osaka University Knowledge Archive : OUKA

https://ir. Library. osaka-u. ac. jp/

Osaka University 


\title{
Image reconstruction for thin observation module by bound optics by using the iterative backprojection method
}

\author{
Kouichi Nitta, Rui Shogenji, Shigehiro Miyatake, and Jun Tanida
}

\begin{abstract}
A method for reconstructing high-spatial-resolution images in an imaging system known as thin observation module by bound optics (TOMBO) is reported. We investigate a novel procedure combining a pixel-rearrangement method and iterative backprojection (IBP). Pixel rearrangement has been used until now in TOMBO, and IBP is a digital superresolution technique. We verify the effectiveness of the combined procedure with simulated and experimental results. (C) 2006 Optical Society of America

OCIS codes: $\quad 110.2970,110.4190,100.3010,100.3020,230.0250,230.3120$.
\end{abstract}

\section{Introduction}

Recently, owing to the remarkable progress made in the fields of solid-state imaging devices, data storage, and image processing, the adoption of digital imagecapturing systems has grown dramatically, and they are now in widespread use around the world. This, in turn, has led to significant expansion of the field of digital imaging. Such systems are considered to be a key technology in the future. To accelerate the ubiquitous adoption of these technologies, for example, in portable information terminals, more compact and lightweight imaging systems are required. However, current compact imaging systems such as microlenses have insufficient imaging quality, and therefore improved compact optical components, in addition to novel system architectures, will be required to meet these demands.

A system architecture known as thin observation module by bound optics (TOMBO) was proposed to construct compact, low-profile image-capturing devices. ${ }^{1}$ This system consists of an optical module

K. Nitta (nitta@kobe-u.ac.jp) is with the Faculty of Engineering, Kobe University, 1-1 Rokkodai-cho, Nada, Kobe 657-8501, Japan. R. Shogenji and J. Tanida are with the Graduate School of Information Science and Technology, Osaka University, 2-1 Yamadaoka, Suita, Osaka 565-0871, Japan. S. Miyatake is with Konica Minolta Technology Center, Inc., 1-2 Sakura-machi, Takatsuki, Osaka 569-5803, Japan.

Received 12 August 2005; revised 9 January 2006; accepted 10 January 2006; posted 13 January 2006 (Doc. ID 64076).

0003-6935/06/132893-08\$15.00/0

(C) 2006 Optical Society of America that uses compound-eye imaging technology and digital signal processing for image reconstruction. Compound-eye imaging imitates the visual organs of some arthropods. ${ }^{2-4}$ One of the attractive features of this system is that the optical module can be made quite thin. Another is that digital signal processing can be used to improve the quality of the image captured by the optical module.

Various issues were identified to improve the performance of the TOMBO system. In terms of the optical hardware, high imaging performance of each microlens, uniformity of the lens array, and accurate alignment between the lens array and the imaging device are required. On the other hand, the digital signal processing should be capable of retrieving detailed information about the target object. In particular, restoration of the high-frequency components in the target image is one of the most important issues. The pixel rearrangement method presented in Ref. 5 is not, by itself, an effective solution for this problem, even though the method is very useful for compensation of misalignment in the optical module.

In this paper, therefore, we present a novel method for image reconstruction in the TOMBO system. In this method the pixel-rearrangement method and iterative backprojection (IBP) ${ }^{6}$ are used complementarily. IBP is a digital superresolution technique that can generate an image with high spatial resolution from a set of images with low spatial resolution. We demonstrate with simulated and experimental results that the proposed method improves the spatial resolution of the reconstructed image. 


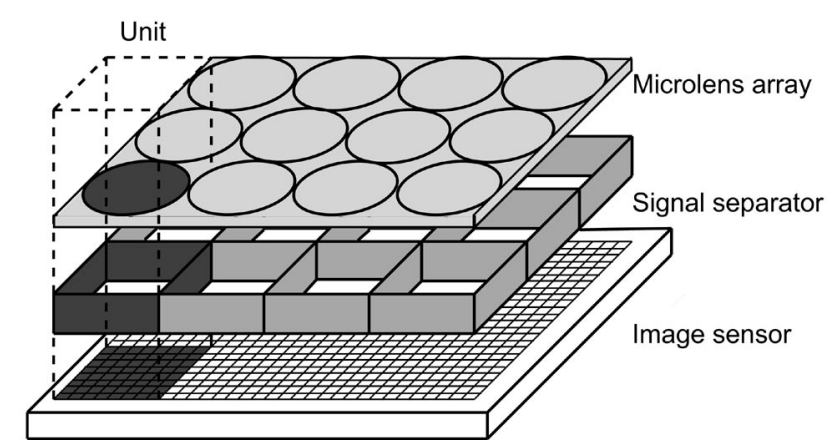

Fig. 1. Schematic diagram of optical module in the TOMBO system.

\section{Thin Observation Module by Bound Optics}

\section{A. Architecture}

The TOMBO system consists of an optical module that uses compound-eye imaging and electronic signal processing for image reconstruction. In the optical module a set of images with low spatial resolution are captured simultaneously to form a single image called a compound image. The signal processing then converts the compound image to a single image with high spatial resolution.

Figure 1 shows a schematic diagram of the optical module in the TOMBO system. Components of the optical module are a microlens array, a signal separator, and a photodetector array. As shown in Fig. 1 , an elemental optical system used to acquire one low-spatial-resolution image is called a unit, and the image obtained is called a unit image. The optical module is a collection of these units, and the set of unit images forms the compound image. Since this kind of compound-eye imaging system acquires visual information with a set of small lenses, a compact, lightweight imaging system can be constructed.

In the visual organs of certain arthropods, an optical signal obtained by a single lens is detected by a single detector. On the other hand, TOMBO has flexibility in terms of the number of photodetectors used per lens system. The number of the photodetectors $N$, the number of units $\mu$, and the number of pixels in a single unit $v$ are the system parameters. The relation among these three parameters is represented by

$$
\nu=N / \mu
$$

The number of pixels in a single unit corresponds directly to the aperture size of the imaging lens and the working distance. Therefore the larger the value of $\mu$, the thinner the system. In particular, in the case of $\mu=N$, the system is equivalent in architecture to the arthropod vision system mentioned above.

The functionality of the TOMBO system can be expanded by modifying the architecture of the optical module and the digital processing. In Ref. 7 two methods for color imaging were reported. One is color separation by pixels, which is used in commercial

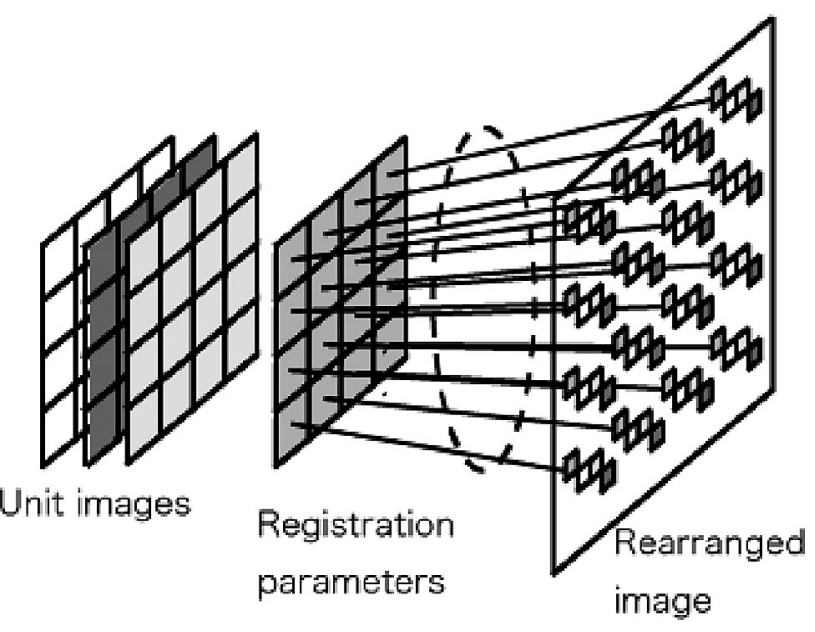

Fig. 2. Schematic diagram of the pixel-rearrangement method.

image sensors, and the other is color separation by units, which gives superior multispectral imaging performance. ${ }^{8}$ Also, fingerprint capturing has been reported as an application of TOMBO. ${ }^{9}$

\section{B. Pixel-Rearrangement Method}

As the signal processing used for image reconstruction in TOMBO, a pixel-sampling method and a pseudomatrix method were studied in Ref. 1. However, the quality of the reconstructed images obtained by these methods was not suitable for practical use. The main reasons for the poor image quality were considered to be (1) misalignment between the microlens array and the photodetector array and (2) undersampling due to compound-eye imaging.

In the optical module for the TOMBO system, accurate alignment is required to obtain a reconstructed image with high quality, but slight errors are unavoidable. Also, each unit image has fewer pixels than those of conventional single-eye imaging systems. Thus compound-eye imaging cannot acquire high-frequency components of the target object. Post-signal processing should be capable of compensating for these alignment errors and of achieving operations equivalent to oversampling in order to retrieve the original information.

The pixel-rearrangement method was proposed to solve problem (1) above. Figure 2 shows a schematic diagram of this method. All pixels in the unit images are remapped onto a virtual plane, and the geometric relations between the unit images and the virtual plane are described by registration parameters. In the processing, the relations are described with an affine transformation model, whose parameters are estimated statistically. ${ }^{5}$

The first step of the pixel-rearrangement method is to compensate for the shading effects caused by the limited aperture of the microlenses and spatial variations in the photodetectors. Thus the captured compound image is corrected in intensity. The corrected image is then divided into a set of unit images. The registration parameters are determined based on the 


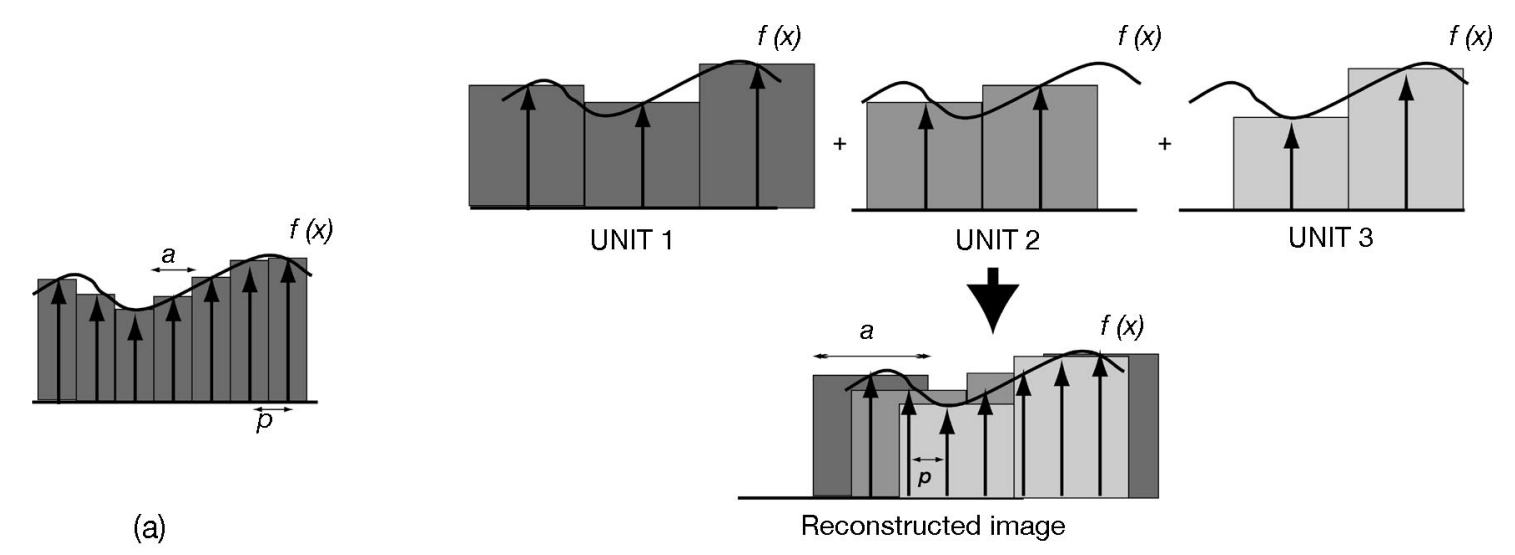

(b)

Fig. 3. One-dimensional model $[g(x)]$ for (a) single-eye imaging and (b) the pixel-rearrangement method in TOMBO.

scheme described above, and the pixels in all unit images are mapped onto the virtual image plane on the basis of the registration parameters. If blank pixels remain in the virtual image, interpolation operations are performed to determine these pixel values.

The pixel-rearrangement method is suitable for high-speed processing because its principle is very simple. Although we confirmed that this method could compensate for misalignment to improve the quality of the output image, ${ }^{5}$ the method cannot resolve the problem caused by undersampling because the pixels in the unit images, which have low spatial resolution, are mapped onto the corresponding pixels on the virtual plane without any oversampling process.

\section{Limitations of the Pixel-Rearrangement Method}

In this section we describe a frequency analysis of the image reconstruction with the pixel-rearrangement method. For this analysis, a simple one-dimensional model that satisfies the sampling theorem is assumed. Figures 3(a) and 3(b) show the models for conventional single-eye imaging and for the pixelrearrangement method used in TOMBO, respectively. In the models the fill factor of the pixels is assumed to be $100 \%$. In this figure $f(x), p$, and $a$ represent the target object, the pixel pitch, and the observation area of a pixel, respectively. In the conventional imaging model the values of $a$ and $p$ are the same. On the other hand, in the TOMBO model the value of $a$ is larger than that of $p$.

In both models, pixel values of an image $g(x)$ are represented by

$$
g(x)=f(x) \operatorname{comb}(x / p) * \operatorname{rect}(x / a)
$$

where $*$ indicates the convolution operator and $\operatorname{comb}(x / p)$ and $\operatorname{rect}(x / a)$ are terms caused by the effects of pixel sampling and the pixel window, respectively.

The Fourier transform of $g(x)$ is represented by

$$
G\left(v_{x}\right)=|a p| F\left(v_{x}\right) * \operatorname{comb}\left(p v_{x}\right) \operatorname{sinc}\left(a v_{x}\right)
$$

where $v_{x}$ is the spatial frequency of the target image. Figure 4 shows profiles of $G\left(v_{x}\right)$ for the two cases. In conventional single-eye imaging, the Nyquist frequency $v_{N}$ is given by

$$
v_{N}=1 / 2 p
$$

From Fig. 4(a), $G\left(v_{x}\right)$ becomes positive for $\left|v_{x}\right|<v_{N}$. This means that the effect of the pixel window in $g(x)$

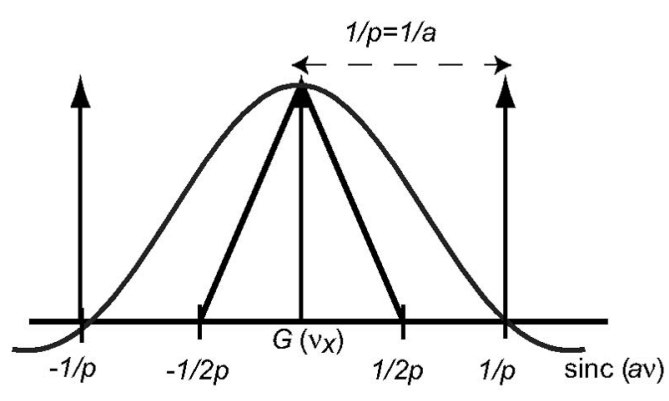

(a)

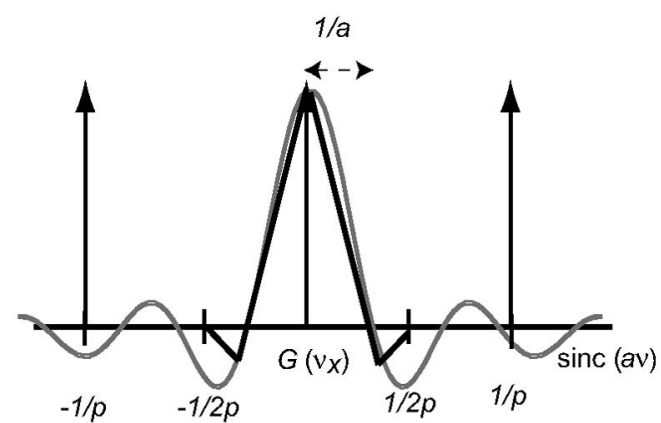

(b)

Fig. 4. $G\left(v_{x}\right)$ of both analysis models: (a) single-eye imaging and (b) the pixel-rearrangement method in TOMBO. 


\section{Inferred image $f^{(n)}$}

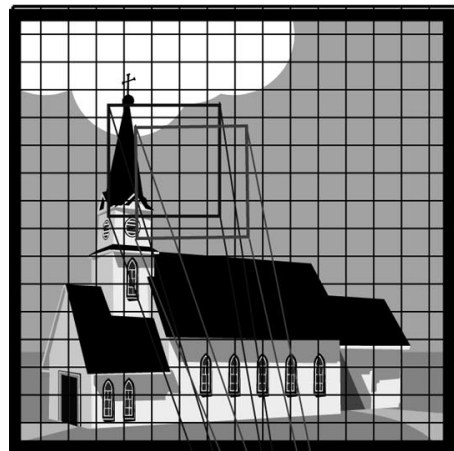

IV process $T u_{X}, u y$

\section{Object $f$ (unknown)}

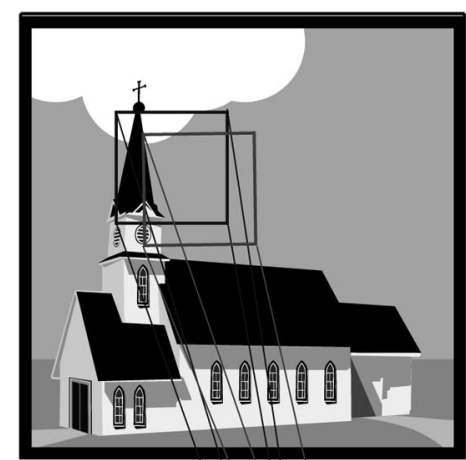

Imaging process

Comparison between simulated and observed low-resolution images

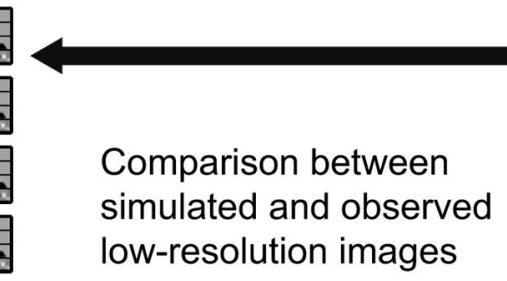

$$
\begin{aligned}
& \text { Simulated } \\
& \text { low-resolution } \\
& \text { images } g_{u_{x}, u_{y}}^{(n)}
\end{aligned}
$$

Fig. 5. Schematic diagram of the IBP method.

can be corrected with simple spatial-frequency filtering. In the case of compound-eye imaging, at $a<2 p$ [as shown in Fig. 4(b)], $G\left(v_{x}\right)$ becomes negative for $1 / a<\left|v_{x}\right|<v_{N}$. This means that the highfrequency components of $f(x)$ are inverted in phase and that the components at the zero-crossing points are lost.

Consequently, it is clear that the TOMBO method based on the pixel-rearrangement method cannot achieve the same performance as conventional single-eye imaging. Therefore we should investigate digital superresolution methods that will be suitable for compound-eye imaging.

\section{Iterative Backprojection Method}

\section{A. Processing Procedure}

We investigated $\mathrm{IBP}^{6,10}$ as a useful digital superresolution algorithm in the TOMBO system. In the IBP method a reconstructed image is generated with an iterative process of error estimation and an inference of a deblurred image. Figures 5 and 6 show a schematic diagram and a flow chart of the IBP method, respectively. In these figures $f$ shows the target object, which is unknown; $f^{(n)}$ is the inferred image gen- erated after $n$ iterations; and $g_{u_{x}, u_{y}}$ and $g_{u_{x}, u_{y}}^{(n)}$ indicate the observed low-resolution image and the simulated one obtained by computation. In the TOMBO system, $g_{u_{x}, u_{y}}$ represents a unit image at position $\left(u_{x}, u_{y}\right)$.

As the first operation of the iterative process, $g_{u_{x} u_{y}}^{(n)}$ are generated by using

$$
g_{u_{x}, u_{y}}^{(n)}=\left[T_{u_{x}, u_{y}}\left(f^{(n)}\right) * h\right] \downarrow s,
$$

where $T_{u_{x}, u_{y}}$ describes a geometric transformation between the target object and the unit at $\left(u_{x}, u_{y}\right)$ and $h$ and $\downarrow s$ represent a blur kernel determined by the lens system and a downsampling operator, respectively.

The next process is estimation of the error function $e^{(n)}$, which is given by

$$
e^{(n)}=\left[\left(1 / N^{2}\right) \sum_{u_{y}=1}^{N} \sum_{u_{x}=1}^{N}\left(g_{u_{x}, u_{y}}-g_{u_{x}, u_{y}}^{(n)}\right)^{2}\right]^{1 / 2} .
$$

If $e^{(n)}$ is less than a threshold value, $f^{(n)}$ is output as the final result and the process is terminated. If $e^{(n)}$ is larger than the threshold value, however, $f^{(n)}$ is updated by using 


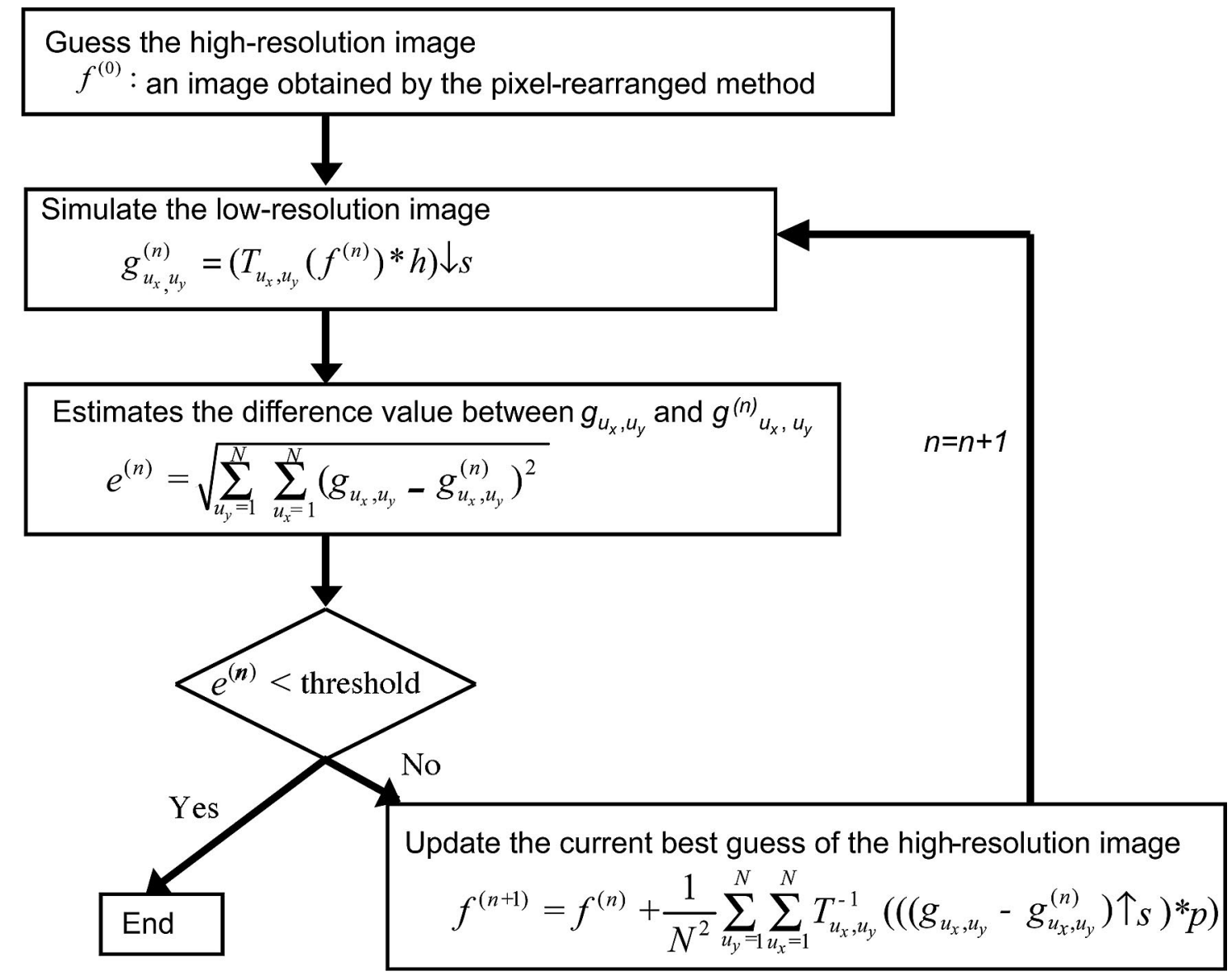

Fig. 6. Flow chart of the IBP method for the TOMBO system.

$$
\begin{aligned}
f^{(n+1)}= & f^{(n)}+\left(1 / N^{2}\right) \sum_{u_{y}=1}^{N} \sum_{u_{x}=1}^{N} T_{u_{x}, u_{y}}^{-1} \\
& \times\left\{\left[\left(g_{\left(u_{x}, u_{y}\right)}-g_{u_{x}, u_{y}}^{(n)}\right) \uparrow s\right] * p\right\},
\end{aligned}
$$

where $p$ and $\uparrow s$ represent the backprojection kernel and an upsampling operator, respectively. When the relation between $h$ and $p$ shown in Eq. (8) is satisfied, $e^{(n)}$ converges in the iterative process ${ }^{10}$ :

$$
\|\delta-h * p\|_{2} \leq 1 \text {. }
$$

B. Combined Procedure: Iterative Backprojection and Pixel-Rearrangement Methods

When the IBP method is applied to a compound image captured by the TOMBO system, a noise signal caused by the misalignment prevents the desired superresolution effect. The reason is that the superresolution processing often emphasizes the noise components in $f^{(n)}$. Therefore here we consider a combination of the pixel-rearrangement method and the IBP method to construct a practical procedure for image reconstruction.

The pixel-rearrangement method is useful for compensation of misalignment, whereas the IBP method is effective for signal retrieval of highspatial-frequency components. One of the most simple but effective approaches is to apply both methods in

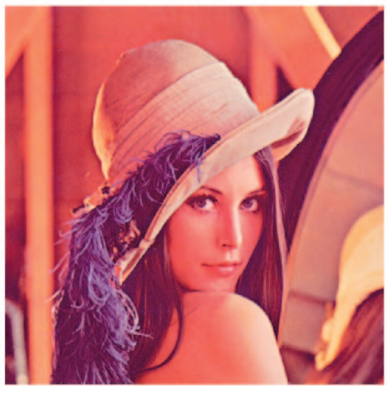

(a)

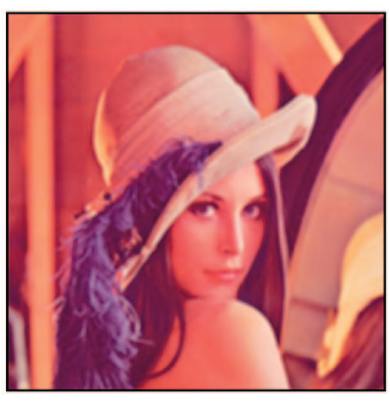

(c)

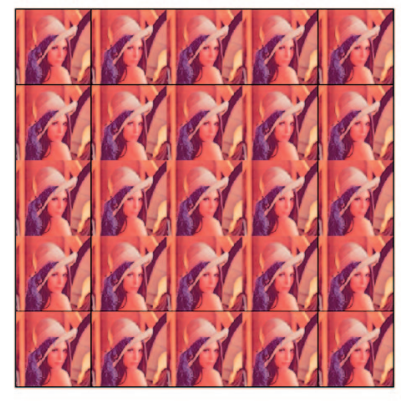

(b)

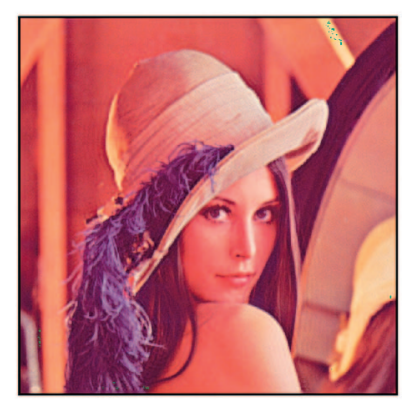

(d)
Fig. 7. (Color online) Simulation results: (a) the original image, (b) the compound image, (c) the result obtained by the pixelrearrangement method, (d) the result of the IBP method after 2500 iterations. 


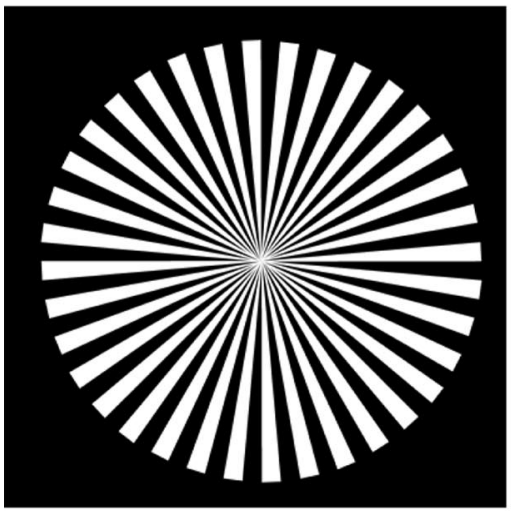

(a)

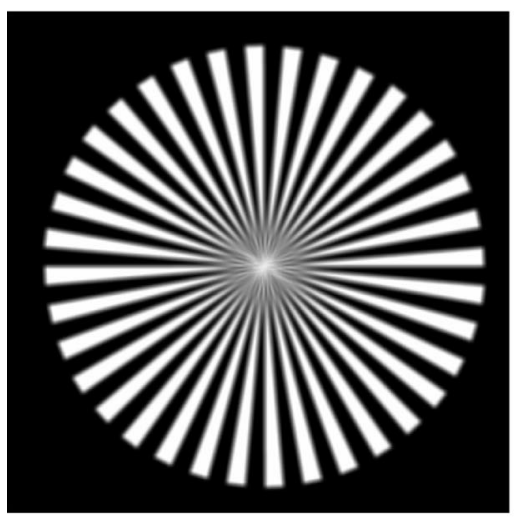

(c)

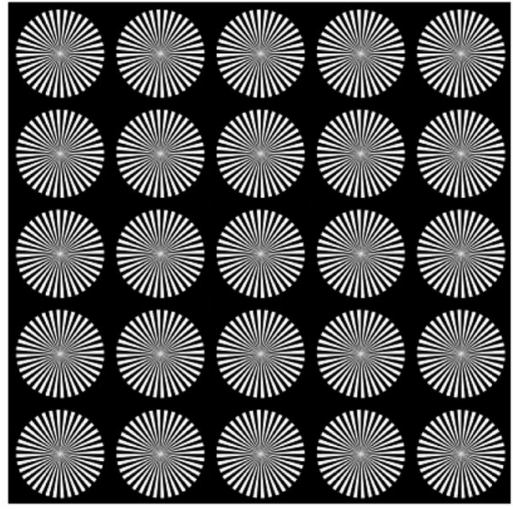

(b)

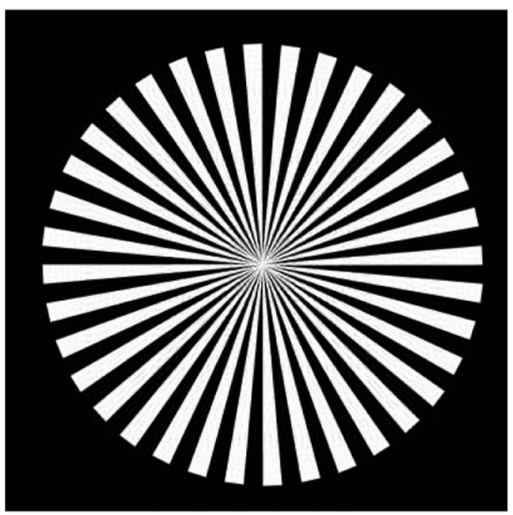

(d)
Fig. 8. Simulation results with a resolution chart: (a) the original image, (b) the compound image, (c) the result obtained by the pixelrearrangement method, (d) the result of the IBP method after 2500 iterations. sequence. First, the pixel-rearrangement method is applied to the image captured by the optical module of the TOMBO system. Then the interpolated image obtained by the pixel-rearrangement method is assigned as the initial image $f^{(0)}$ for the IBP method, and the iterative process is started. After $n$ iterations, $f^{(n)}$ is output as the final result.

In our experimental results, described in Section 5 , an explicit threshold value of the IBP method is not set. Instead, the number of iterations is set manually. Also, in red-green-blue (RGB) color imaging, the above procedure is applied to the individual color channels independently.

\section{Experimental Verification}

\section{A. Simulation Results}

First, we verified the effectiveness of our proposed image reconstruction method by simulation. Figure 7 shows the simulation results. The $\nu$ and $\mu$ parameters for the TOMBO system were assumed to be 5 and 80 , respectively. Figure 7 (a) shows the original RGB color image with $400 \times 400$ pixels, and Fig. 7(b) shows the compound image. The reconstructed image obtained by the pixel-rearrangement method is shown in Fig. 7(c). Because $5 \times 5$ units were used, the number of pixels in the image was $400 \times 400$. Figure $7(\mathrm{~d})$ shows the results obtained with the proposed procedure after 200 iterations. In comparison with
Fig. 7(c), the obtained results appear sharper, particularly in the edge regions, where the sharpening effect is more noticeable.

In a subsequent simulation a resolution chart was used to investigate the effectiveness of the proposed method in terms of restoration of high-spatialfrequency components. In this simulation the values of $\mu$ and $v$ were equivalent to those in the above simulation. Figures 8(a) and 8(b) show the test chart and the simulated compound image. The reconstructed image obtained by the pixel-rearrangement method is shown in Fig. 8(c). The central part of Fig. 8(c) is blurred in comparison with that in the original chart. The reasons for this were described in Section 3 above. Figure 8 (d) shows the image obtained by the proposed method after 2500 iterations. From this image, we have verified that the iterative processing in the IBP method achieves the desired image restoration.

\section{B. Experimental Results}

The procedure was experimentally applied to an image obtained by a prototype of the TOMBO system. ${ }^{11}$ Figure 9 shows a photograph of the sensor module. In the two-dimensional (2D) lens array (Advanced Microoptic Systems, GmbH) $10 \times 10$ lenses are aligned with a pitch of $0.5 \mathrm{~mm}$. The focal length and the diameter of each lens were 1.3 and $0.5 \mathrm{~mm}$, respectively. A signal separator, formed of a grid of stainless steel plates with a thickness of $50 \mu \mathrm{m}$, was inserted 


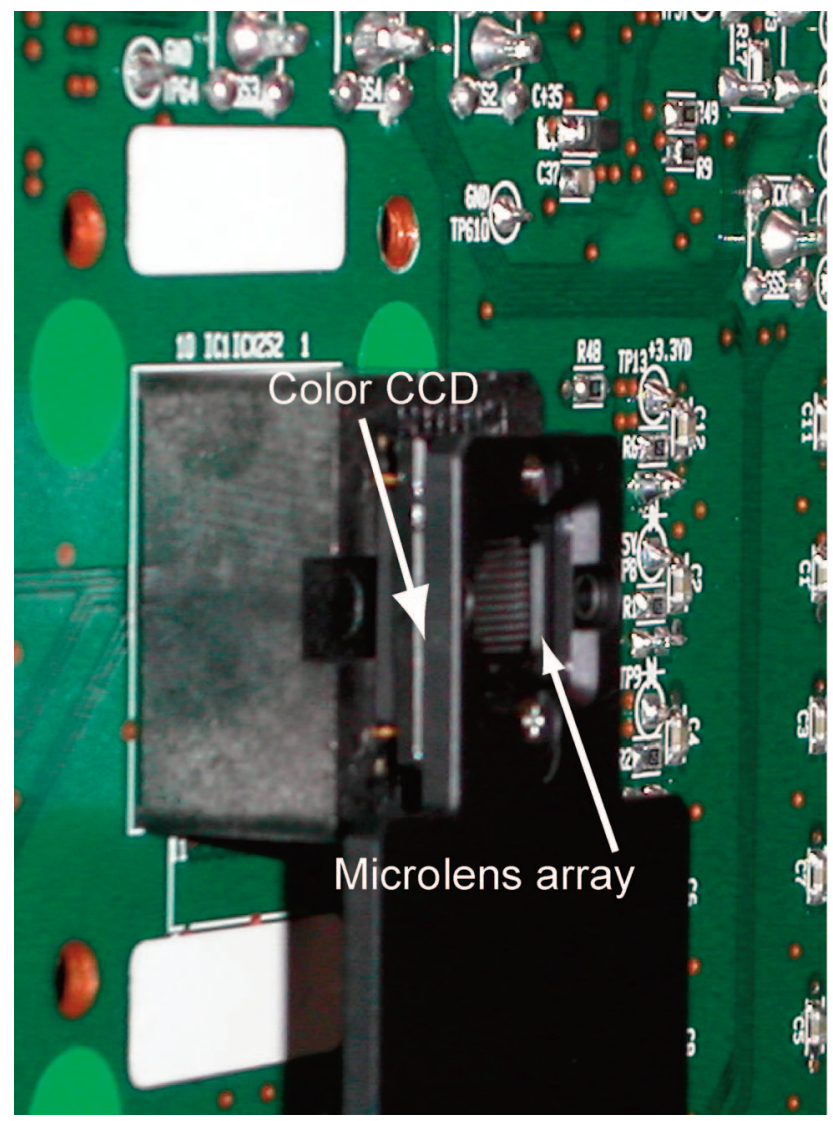

Fig. 9. (Color online) Picture of the prototype module.

between the lens array and the image sensor. For the image sensor, a commercial color CCD with a pixel pitch and pixel size of $3.125 \mu \mathrm{m}$ was used. A Bayer color filter was attached to the sensor. This setup allowed RGB images with $80 \times 80$ pixels $(v=80)$ to be obtained from each unit.

Figure 10 shows the experimental results. The distance between the target object and the microlens array was $\sim 520 \mathrm{~mm}$. Over this distance, $5 \times 5$ units of the prototype module uniformly captured information on the target. Figures 10(a) and 10(b) show the reconstructed image obtained by the pixelrearrangement method and that obtained by the IBP method, respectively. Both images have $400 \times 400$ pixels, formed by the $5 \times 5$ unit images.

Figures 10(c) and 10(d) depict a specific part extracted from Figs. 10(a) and 10(b). In comparison with these images, it is clear that the IBP method is more effective for the central part of the reconstructed image. However, the resolution at the marginal parts of Fig. 10(b) is not improved. In Fig. 10(b) the number of iterations was set to 13 so as not to amplify the noise component. In the IBP process $e^{(n)}$ did not converge and reached the minimum value after 13 iterations.

\section{Discussion}

To discuss the effectiveness of the proposed method quantitatively, we estimated the signal-to-noise ratio

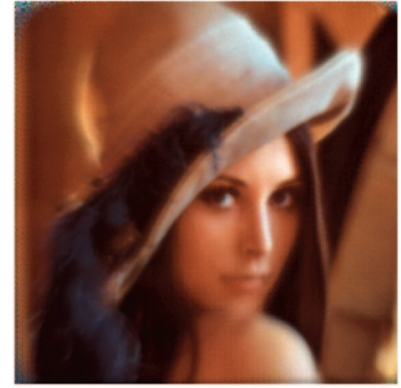

(a)

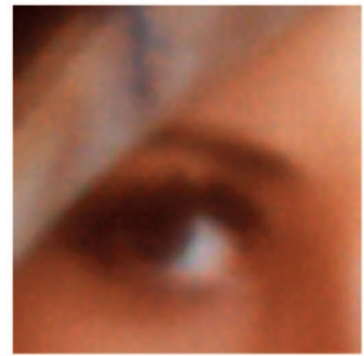

(c)

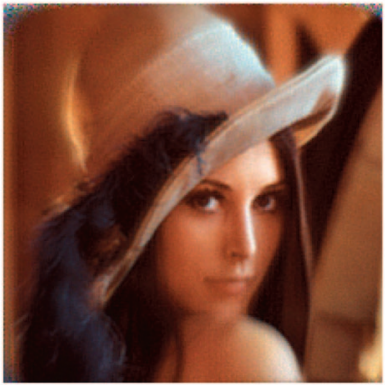

(b)

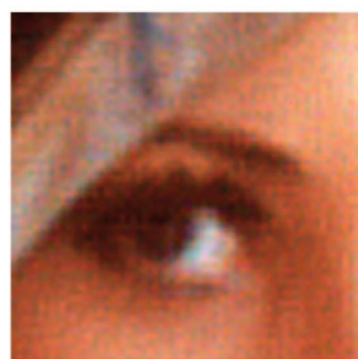

(d)
Fig. 10. (Color online) Experimental results: (a) the reconstructed image with the pixel-rearrangement method and (b) that with the IBP method. (c), (d) Show specific parts of (a) and (b), respectively.

(SNR) between the luminance component of the original image shown in Fig. 7(a) and that of the results obtained by the proposed method. Figure 11 shows the relation between the SNR and the number of iterations $n$. At $n=0$, the output image is equivalent to the result obtained by the pixel-rearrangement method. In this case the SNR is $\sim 28.1 \mathrm{~dB}$. On the other hand, the SNR is improved to $34.5 \mathrm{~dB}$ for $n=200$. Therefore it is confirmed that the IBP method is useful for image restoration.

The result reconstructed from an experimental compound image is now discussed. As shown in Fig 10(b), improvement of the resolution is weak at the marginal parts of the reconstructed image. This is caused by the difference in image quality between the center and the marginal parts in each unit. The blur kernel $h$ and the backprojection kernel $p$ should therefore be optimized per pixel to resolve this problem; this issue will be dealt with in a future study.

The presence of slight errors in the registration parameters is considered to be one of the reasons why $e^{(n)}$ does not converge. In the iterative process of the IBP method, this error will be amplified as a noise component. Therefore we should determine the registration parameters more accurately in the pixelrearrangement method to improve the effectiveness of our procedure.

\section{Conclusion}

In this paper we have investigated a method for image reconstruction based on digital superresolution that is suitable for the TOMBO system. Using both the IBP method and the pixel-rearrangement 


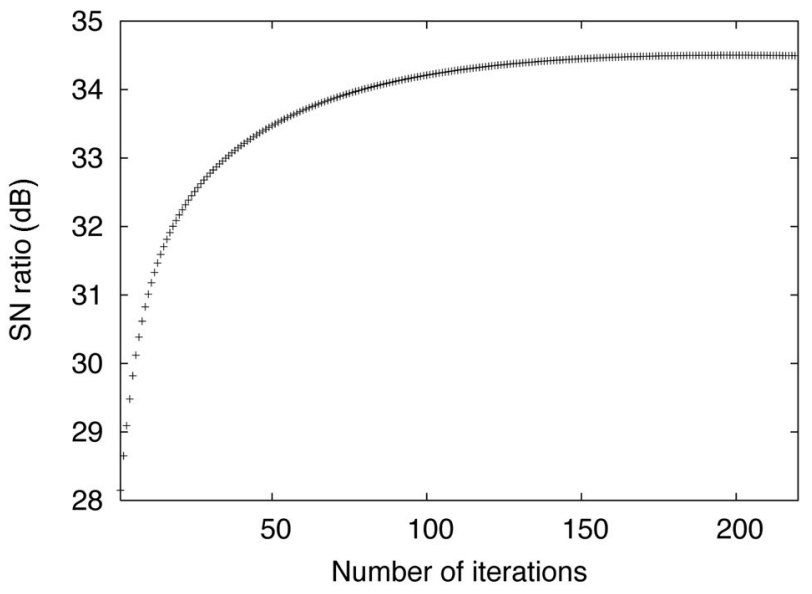

Fig. 11. Relation between SNR and the number of iterations $n$.

method, we have constructed a useful procedure to restore high-spatial-resolution information. The effectiveness of the procedure has been shown by experimental and simulation results. In particular, in the simulation it was verified that the proposed procedure achieves a SNR that is $6.4 \mathrm{~dB}$ higher than that achieved by the pixel-rearrangement method. In future research, to improve the image quality of the final result, we should improve the accuracy of the process for shading compensation and the determination of registration parameters in the pixel-rearrangement method.

This research was supported by the Japan Science and Technology Agency under the UltraThin Image Capturing Module of the Regional Science Promotion Program. We also thank Sony Semiconductor Kyusyu Co. for providing the CCD image sensors.

\section{References}

1. J. Tanida, T. Kumagai, K. Yamada, S. Miyatake, K. Ishida, T. Morimoto, N. Kondou, D. Miyazaki, and Y. Ichioka, "Thin observation module by bound optics (TOMBO): concept and experimental verification,” Appl. Opt. 40, 1806-1813 (2001).

2. S. Ogata, J. Ishida, and T. Sasano, "Optical sensor array in an artificial compound eye,” Opt. Eng. 33, 3649-3655 (1994).

3. J. S. Sanders and C. E. Halford, "Design and analysis of apposition compound eye optical sensors," Opt. Eng. 34, 222-235 (1995).

4. K. Hamanaka and H. Koshi, "An artificial compound eye using a microlens array and its application to scale-invariant processing," Opt. Rev. 3, 264-268 (1996).

5. Y. Kitamura, R. Shogenji, K. Yamada, S. Miyatake, M. Miyamoto, T. Morimoto, Y. Masaki, N. Kondou, D. Miyazaki, J. Tanida, and Y. Ichioka, "Reconstruction of a high-resolution image on a compound-eye image-capturing system," Appl. Opt. 43, 1719-1727 (2004).

6. M. Irani and S. Peleg, "Improving resolution by image registration," CVGIP Graph. Models Image Process. 53, 231-239 (1991).

7. J. Tanida, R. Shogenji, Y. Kitamura, K. Yamada, M. Miyamoto, and S. Miyatake, "Color imaging with an integrated compound imaging system," Opt. Express 11, 18-26 (2003).

8. R. Shogenji, Y. Kitamura, K. Yamada, S. Miyatake, and J. Tanida, "Multispectral imaging using compact compound optics," Opt. Express 12, 1643-1655 (2004).

9. R. Shogenji, Y. Kitamura, K. Yamada, S. Miyatake, and J. Tanida, "Bimodal fingerprint capturing system based on compound-eye imaging module," Appl. Opt. 43, 1355-1359 (2004).

10. M. Irani and S. Peleg, "Motion analysis for image enhancement: resolution, occlusion, and transparency," J. Visual Commun. Image Represent. 4, 324-335 (1993).

11. J. Tanida, R. Shogenji, Y. Kitamura, K. Yamada, M. Miyamoto, and S. Miyatake "Functional extension of thin observation module by bound optics (TOMBO)," in Optics in Computing, vol. 90 of OSA Trends in Optics and Photonics Series (Optical Society of America, 2003), pp. 170-172 (2003). 